\begin{tabular}{|c|l|}
\hline Title & Optical characterization of platinum-halide ladder compounds \\
\hline Author(s) & Yamamoto, Shoji; Ohara, Jun \\
\hline Citation & $\begin{array}{l}\text { Physical Review B, 76(23), 235116 } \\
\text { https://doi.org/10.1103/PhysRevB.76.235116 }\end{array}$ \\
\hline Issue Date & 2007 \\
\hline Doc URL & http://hdl.handle.net/2115/32339 \\
\hline Rights & ○2007 The A merican Physical Society \\
\hline Type & article \\
\hline File Information & PhysRevB_76_235116.pdf \\
\hline
\end{tabular}

Instructions for use 


\title{
Optical characterization of platinum-halide ladder compounds
}

\author{
Shoji Yamamoto and Jun Ohara \\ Department of Physics, Hokkaido University, Sapporo 060-0810, Japan \\ (Received 18 June 2007; revised manuscript received 24 July 2007; published 14 December 2007)
}

\begin{abstract}
Varieties of quasi-one-dimensional halogen $(X)$-bridged transition-metal $(M)$ complexes, $\left(\mathrm{C}_{8} \mathrm{H}_{6} \mathrm{~N}_{4}\right)$ $\times\left[\mathrm{Pt}\left(\mathrm{C}_{2} \mathrm{H}_{8} \mathrm{~N}_{2}\right) X\right]_{2} X\left(\mathrm{ClO}_{4}\right)_{3} \cdot \mathrm{H}_{2} \mathrm{O}(X=\mathrm{Br}, \mathrm{Cl})$ and $\left(\mathrm{C}_{10} \mathrm{H}_{8} \mathrm{~N}_{2}\right)\left[\mathrm{Pt}\left(\mathrm{C}_{4} \mathrm{H}_{13} \mathrm{~N}_{3}\right) \mathrm{Br}_{2} \mathrm{Br}_{4} \cdot 2 \mathrm{H}_{2} \mathrm{O}\right.$, comprising two-leg ladders of mixed-valent platinum ions, are described in terms of a multiband extended Peierls-Hubbard Hamiltonian. The polarized optical-conductivity spectra are theoretically reproduced, and the ground-state valence distributions are reasonably determined. The latter variety, whose interchain valence arrangement is out of phase, is reminiscent of conventional $M X$ single-chain compounds, while the former variety, whose interchain valence arrangement is in phase, reveals itself as a $d$ - $p$ - $\pi$-hybridized multiband ladder material.
\end{abstract}

DOI: $10.1103 /$ PhysRevB.76.235116

PACS number(s): 71.20.Be, 71.45.Lr, 78.20.Ci, 78.20.Bh

\section{INTRODUCTION}

Quasi-one-dimensional transition-metal $(M)$ complexes with bridging halogens ${ }^{1-4}(X)$ have been attracting much interest for several decades, and significant efforts are still devoted in fabricating their new varieties. Conventional platinum-halide chains exhibit a Peierls-distorted mixedvalent ground state, ${ }^{5}$ while their nickel analogs have a Mottinsulating monovalent regular-chain structure., ${ }^{6,7}$ Palladiumhalide chains are intermediates with a ground state tunable optically ${ }^{8,9}$ and electrochemically. ${ }^{10,11}$ The chargedensity-wave (CDW) ground state can be tuned by halogen doping $^{12-14}$ and pressure application ${ }^{15}$ as well. Metal binucleation leads to a wider variety of electronic states. ${ }^{16,17}$ A diplatinum iodide chain compound, $\left[\left(\mathrm{C}_{2} \mathrm{H}_{5}\right)_{2} \mathrm{NH}_{2}\right]_{4}\left[\mathrm{Pt}_{2}\left(\mathrm{P}_{2} \mathrm{O}_{5} \mathrm{H}_{2}\right)_{4} \mathrm{I}\right]$, exhibits photo- and pressure-induced phase transitions, ${ }^{18-22}$ whereas its analog without any counter ion, $\mathrm{Pt}_{2}\left(\mathrm{CH}_{3} \mathrm{CS}_{2}\right)_{4} \mathrm{I}$, is of metallic conduction at room temperature and undergoes successive phase transitions ${ }^{23,24}$ with decreasing temperature. There are further attempts $^{25,26}$ to bridge polynuclear and/or heterometallic units by halogens.

More than $300 M X$ compounds have thus been synthesized so far, but their crystal structures are all based on $M X$ single chains. In such circumstances, several authors ${ }^{27,28}$ have recently succeeded in assembling $M X$ complexes within a ladder lattice. Metal oxide ladders are generally remarkable for their strongly correlated $d$ electrons. $\mathrm{SrCu}_{2} \mathrm{O}_{3}$ behaves as a $d$ - $p$ ladder of the Hubbard type, ${ }^{29}$ whereas $\mathrm{NaV}_{2} \mathrm{O}_{5}$ is well describable within a single-band HolsteinHubbard Hamiltonian. ${ }^{30}$ On the other hand, the newly synthesized metal-halide ladders are double featured with competing electron-electron and electron-phonon interactions ${ }^{31}$ and are possibly of $d-p-\pi$-mixed character. Such a multicolored stage potentially exhibits a variety of electronic states, and it is highly interesting to control them chemically and physically. Thus motivated, we make a model study of ladder-shaped $M X$ compounds, ( $\mu$-bpym) $\times[\mathrm{Pt}(\mathrm{en}) X]_{2} X\left(\mathrm{ClO}_{4}\right)_{3} \cdot \mathrm{H}_{2} \mathrm{O} \quad(X=\mathrm{Br}, \mathrm{Cl} ;$ en=ethylendiamine $=\mathrm{C}_{2} \mathrm{H}_{8} \mathrm{~N}_{2} ; \quad \mu$-bpym $=2,2^{\prime}$-bipyrimidine $\left.=\mathrm{C}_{8} \mathrm{H}_{6} \mathrm{~N}_{4}\right) \quad$ and (bpy) $[\mathrm{Pt}(\text { dien }) \mathrm{Br}]_{2} \mathrm{Br}_{4} \cdot 2 \mathrm{H}_{2} \mathrm{O} \quad$ (dien = diethylentriamine $=\mathrm{C}_{4} \mathrm{H}_{13} \mathrm{~N}_{3}$; bpy $=4,4^{\prime}$ - bipyridyl $=\mathrm{C}_{10} \mathrm{H}_{8} \mathrm{~N}_{2}$ ), which are hereafter referred to as (bpym) $[\mathrm{Pt}(\mathrm{en}) \mathrm{X}]_{2}$ and (bpy) $\times[\mathrm{Pt}(\text { dien }) \mathrm{Br}]_{2}$, respectively.

\section{GROUND-STATE PHASE COMPETITION}

Resonant Raman spectra of (bpym) $[\operatorname{Pt}(\text { en }) X]_{2}$ and (bpy) $\times[\mathrm{Pt}(\text { dien }) \mathrm{Br}]_{2}$ both suggest a $\mathrm{Pt}^{2+} / \mathrm{Pt}^{4+}\left[\mathrm{Pt}^{3 \mp} \delta(0<\delta \leqslant 1)\right.$ in practice] valence-alternating ground state. ${ }^{27,28}$ Then, how is the interchain valence arrangement, in phase (IP) or out of phase (OP)? CDW states of the IP and OP types are indeed in close competition with varying interchain electronic communication.

Let us consider a half-filled single-band Hamiltonian,

$$
\begin{aligned}
\mathcal{H}= & \sum_{n=1}^{N} \sum_{l=1}^{2}\left\{K u_{n: l}^{2}-\sum_{s= \pm}\left[\beta\left(u_{n: l}-u_{n-1: l}\right) n_{n: l M s}\right.\right. \\
& \left.+\left(t_{M M}^{\mathrm{leg}} a_{n+1: l M s}^{\dagger} a_{n: l M s}+\frac{t_{M M}^{\mathrm{rung}}}{2} a_{n: l M s}^{\dagger} a_{n: 3-l M s}+\text { H.c. }\right)\right] \\
& +\sum_{s, s^{\prime}= \pm}\left(\frac{\delta_{-s, s^{\prime}}}{2} U_{M} n_{n: l M s} n_{n: l M s^{\prime}}+V_{M M}^{\mathrm{leg}} n_{n: l M s} n_{n+1: l M s^{\prime}}\right. \\
& \left.\left.+\frac{V_{M M}^{\mathrm{rung}}}{2} n_{n: l M s} n_{n: 3-l M s^{\prime}}+V_{M M^{\mathrm{diag}}} n_{n: l M s} n_{n+1: 3-l M s^{\prime}}\right)\right\}
\end{aligned}
$$

assuming the halogen $p_{z}$ and ligand $\pi$ orbitals to be fully filled and thus inactive. Here, except for the intrachain and interchain metal-to-metal supertransfers, $t_{M M}^{\mathrm{leg}}$ and $t_{M M}^{\text {rung }}$, we use the same notation that is later defined in Eq. (3.1) and Fig. 2. The second-order perturbation scheme under the conditions of $t_{M M}^{\text {rung }} \ll t_{M M}^{\text {leg }}$ gives the energies of IP- and OP-CDW states as

$$
\begin{gathered}
\frac{E_{\mathrm{IP}}}{N}=2 K u^{2}-4 \beta u+U_{M}+2 V_{M M}^{\mathrm{rung}}, \\
\frac{E_{\mathrm{OP}}}{N}=2 K u^{2}-4 \beta u+U_{M}+4 V_{M M}^{\mathrm{diag}} \\
-\frac{2\left(t_{M M}^{\mathrm{rung}}\right)^{2}}{4 \beta u-U_{M}+V_{M M}^{\mathrm{rung}}+4\left(V_{M M}^{\mathrm{leg}}-V_{M M}^{\mathrm{diag}}\right)},
\end{gathered}
$$

and they are balanced at 


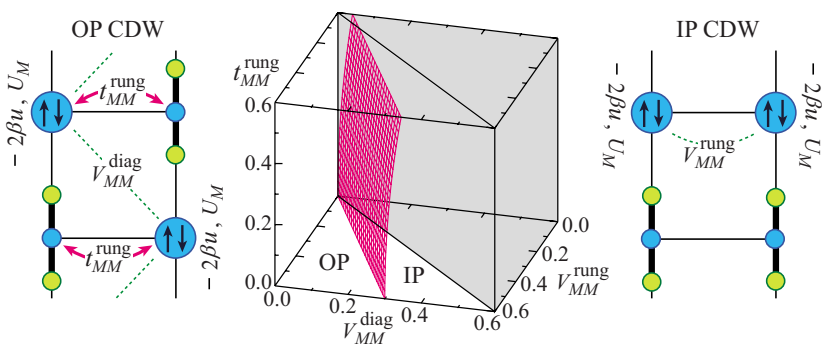

FIG. 1. (Color online) Hartree-Fock calculation of a groundstate phase diagram on the $t_{M M^{\text {rung }}}^{\text {rung }} V_{M M^{-}}^{\text {rung }} V_{M M}^{\text {diag }}$ cube within a singleband model, where $t_{M M}^{\mathrm{leg}}$ is taken as unity and the region of no physical interest is shaded. A simple consideration of competing IP-CDW and OP-CDW states is also presented, where Coulomb energy losses and transfer energy gains within $d$ electrons are illustrated.

$\left(t_{M M}^{\text {rung }}\right)^{2}=\left(2 V_{M M}^{\text {diag }}-V_{M M}^{\text {rung }}\right)\left[4 \beta u-U_{M}+V_{M M}^{\text {rung }}+4\left(V_{M M}^{\text {leg }}-V_{M M}^{\text {diag }}\right)\right]$,

where $u \equiv\left|u_{n: l}\right|$ is the halogen-ion displacement in isolated $M X$ chains. We show in Fig. 1 a numerical phase diagram based on Hamiltonian (2.1), which agrees well to the estimate (2.4). IP CDW and OP CDW are stabilized with increasing $V_{M M}^{\mathrm{diag}}$ and $V_{M M}^{\mathrm{rung}}$, respectively. OP CDW is further stabilized with increasing $t_{M M}^{\text {rung }}$, while IP CDW has no chance of interchain electron transfer without $\pi$ orbitals mediation (in the strongly valence-trapped limit, strictly). Nonvanishing optical absorption in the rung direction with the IP-CDW background should be significant of contributive ligand $\pi$ orbitals.

While the ground-state phase diagram remains almost unchanged with $p$ and/or $\pi$ electrons taken into calculation, the single-band model totally fails to interpret the optical properties. The optical- conductivity spectra measured on (bpym) $[\mathrm{Pt}(\mathrm{en}) \mathrm{X}]_{2}$ and (bpy) $[\mathrm{Pt}(\text { dien }) \mathrm{Br}]_{2}$ are considerably different from each other, but it cannot distinguish between them at all. We proceed to much more elaborate calculations.

\section{MODEL HAMILTONIAN}

We consider a multiband extended Peierls-Hubbard Hamiltonian of $4 / 5$ electron filling on the two-leg ladder lattice,

$$
\begin{aligned}
\mathcal{H}= & \sum_{n, l, s}\left\{\left[\varepsilon_{M}-\beta\left(u_{n: l}-u_{n-1: l}\right)\right] n_{n: l M s}+\varepsilon_{X} n_{n: l X s}+\frac{\varepsilon_{L}}{2} n_{n: L s}\right\} \\
& -\sum_{n, l, s}\left[\left(t_{M X}+\alpha u_{n: l}\right) a_{n+1: l M s}^{\dagger} a_{n: l X s}\right. \\
& +\left(t_{M X}-\alpha u_{n: l}\right) a_{n: l X s}^{\dagger} a_{n: l M s}+t_{M L} a_{n: l M s}^{\dagger} a_{n: L s}+\left(t_{X L}+\gamma u_{n: l}\right) \\
& \left.\times a_{n+1: L s}^{\dagger} a_{n: X s}+\left(t_{X L}-\gamma u_{n: l}\right) a_{n: l X s}^{\dagger} a_{n: L s}+\text { H.c. }\right] \\
& +\sum_{n, l, s, s^{\prime}}\left\{\frac{\delta_{-s, s^{\prime}}}{2}\left(\sum_{A=M, X} U_{A} n_{n: l A s} n_{n: l A s^{\prime}}+\frac{U_{L}}{2} n_{n: L s} n_{n: L s^{\prime}}\right)\right. \\
& +V_{M X}^{\mathrm{leg}}\left(n_{n+1: l M s}+n_{n: l M s}\right) n_{n: l X s^{\prime}}+V_{M L^{\mathrm{rung}}} n_{n: l M s} n_{n: L s^{\prime}}
\end{aligned}
$$

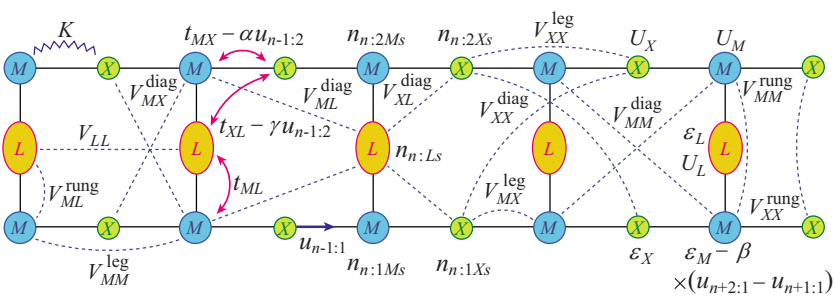

FIG. 2. (Color online) Modeling of $M X$ ladders, where $M=\mathrm{Pt}$; $X=\mathrm{Br}, \mathrm{Cl}$; and $L=\mu$-bpym, bpy. An electron with spin $s=\uparrow, \downarrow \equiv \pm$ is created on the $M d_{z^{2}}$ and $X p_{z}$ orbitals on the $l$ th leg in the $n$th unit by $a_{n: l M s}^{\dagger}$ and $a_{n: I X s}^{\dagger}$, respectively, and on the $n$ th-rung $L \pi$ orbital by $a_{n: L s}^{\dagger}$. The resultant electron density is given by $a_{n: l M s}^{\dagger} a_{n: l M s}$ $\equiv n_{n: I M s}, a_{n: I X s}^{\dagger} a_{n: l X s} \equiv n_{n: I X s}$, and $a_{n: L s}^{\dagger} a_{n: L s} \equiv n_{n: L s}$. The on-site energies (electron affinities) of isolated atoms and molecules are given by $\varepsilon_{A}(A=M, X, L)$, and the electron hoppings between these levels are modeled by $t_{A A^{\prime}}\left[A=M, X ; A^{\prime}(\neq A)=X, L\right] . \quad U_{A}$ and $V_{A A^{\prime}}$ $\left(A, A^{\prime}=M, X, L\right)$ describe the on-site and different-site Coulomb interactions, respectively. The halogen-ion displacements $u_{n: l}$ affect electrons through intersite $(\alpha, \gamma)$ and intrasite $(\beta)$ coupling constants, accompanied by elastic energy $\propto K$.

$$
\begin{aligned}
& +V_{X L}^{\mathrm{diag}} n_{n: l X s}\left(n_{n: L s^{\prime}}+n_{n+1: L s^{\prime}}\right)+V_{M L}^{\mathrm{diag}}\left(n_{n+1: l M s} n_{n: L s^{\prime}}\right. \\
& \left.+n_{n: l M s} n_{n+1: L s^{\prime}}\right)+V_{M X}^{\mathrm{diag}}\left(n_{n+1: l M s}+n_{n: l M s}\right) n_{n: 3-l X s^{\prime}} \\
& +\sum_{A=M, X}\left(V_{A A}^{\mathrm{leg}} n_{n+1: l A s} n_{n: l A s^{\prime}}+\frac{V_{A A}^{\mathrm{rung}}}{2} n_{n: l A s} n_{n: 3-l A s^{\prime}}\right. \\
& \left.\left.+V_{A A}^{\mathrm{diag}} n_{n+1: l A s} n_{n: 3-l A s^{\prime}}\right)+\frac{V_{L L}}{2} n_{n+1: L s} n_{n: L s^{\prime}}\right\}+K \sum_{n, l} u_{n: l}^{2},
\end{aligned}
$$

as is illustrated with Fig. 2, where platinum $d_{z^{2}}$, halogen $p_{z}$, and rung-ligand $\pi$ orbitals are explicitly taken into calculation. For platinum-halide single-chain compounds, typical Pt- $X$ stretching modes are observed with frequencies of $10-40 \mathrm{meV}$ and their coupling strength is estimated to be 2 to $3 \mathrm{eV} / \AA{ }^{32}$ Assuming the in-chain vibrational modes to remain valid in our ladder materials, the phonon energy is less than $1 \%$ of the optical gap $E_{\mathrm{CT}}$ and at most $4 \%$ of the electron-phonon interaction $\beta u$ (see Tables I and II later on). That is why we stand on the adiabatic Hamiltonian (3.1). Such a classical treatment of phonons is widely adopted and generally successful for mixed-valent platinum-halide compounds. ${ }^{3,16,17,31,33-36}$ Quantum phonons may be relatively effective in strongly correlated valence-delocalized nickelhalide chains. ${ }^{4}$

Characterization of the brand new $M X$ ladders is still in the early stage from both experimental ${ }^{27,28}$ and theoretical ${ }^{31,37}$ points of view, and therefore, little is known about the model parameters. In such circumstances, extensive two-band model studies on $M X$ single chains serve as guides to our exploration.

Since the hopping integral $t_{M X}$ is particularly important as an energy scale, we first set it closely consistent with the authorized estimates obtained by the Los Alamos National Laboratory working team. Comparing two-band model descriptions with first-principles local-density-approximation 
TABLE I. Model parameters for (A) (bpym) $[\mathrm{Pt}(\mathrm{en}) \mathrm{Cl}]_{2}$, (B) (bpym) $[\mathrm{Pt}(\mathrm{en}) \mathrm{Br}]_{2}$, and (C) (bpy) $\left[\mathrm{Pt}(\text { dien) } \mathrm{Br}]_{2}\right.$, where $\varepsilon_{M}$ is set equal to zero.

\begin{tabular}{cccccccccccccccc}
\hline \hline & $\mathrm{A}$ & $\mathrm{B}$ & $\mathrm{C}$ & & $\mathrm{A}$ & $\mathrm{B}$ & $\mathrm{C}$ & & $\mathrm{A}$ & $\mathrm{B}$ & $\mathrm{C}$ & & $\mathrm{A}$ & $\mathrm{B}$ & $\mathrm{C}$ \\
\hline$t_{M X}(\mathrm{eV})$ & 1.54 & 1.35 & 1.32 & $V_{M X}^{\text {leg }}(\mathrm{eV})$ & 0.80 & 0.92 & 0.86 & $V_{M X}^{\text {diag }}(\mathrm{eV})$ & 0.22 & 0.19 & 0.14 & $\varepsilon_{X}(\mathrm{eV})$ & -3.39 & -2.43 & -2.38 \\
$t_{M L}(\mathrm{eV})$ & 1.08 & 0.94 & 0.53 & $V_{M M}^{\text {leg }}(\mathrm{eV})$ & 0.23 & 0.20 & 0.20 & $V_{M M}^{\text {diag }}(\mathrm{eV})$ & 0.20 & 0.16 & 0.07 & $\varepsilon_{L}(\mathrm{eV})$ & -0.62 & -0.54 & -0.66 \\
$t_{X L}(\mathrm{eV})$ & 0.15 & 0.13 & 0.05 & $V_{X X}^{\text {leg }}(\mathrm{eV})$ & 0.18 & 0.16 & 0.14 & $V_{X X}^{\text {diag }}(\mathrm{eV})$ & 0.14 & 0.12 & 0.08 & $\beta(e \mathrm{~V} / \AA)$ & 2.37 & 2.40 & 2.27 \\
$U_{M}(\mathrm{eV})$ & 1.23 & 1.08 & 0.92 & $V_{M L}^{\text {rung }}(\mathrm{eV})$ & 0.38 & 0.35 & 0.30 & $V_{M L}^{\text {diag }}(\mathrm{eV})$ & 0.18 & 0.13 & 0.08 & $\alpha(e \mathrm{~V} / \AA)$ & 0.85 & 0.77 & 0.65 \\
$U_{X}(\mathrm{eV})$ & 1.69 & 0.94 & 1.06 & $V_{M M}^{\text {rung }}(\mathrm{eV})$ & 0.23 & 0.20 & 0.16 & $V_{X L}^{\text {diag }}(\mathrm{eV})$ & 0.25 & 0.23 & 0.20 & $\gamma(e \mathrm{~V} / \AA)$ & 0.30 & 0.26 & 0.13 \\
$U_{L}(\mathrm{eV})$ & 1.08 & 0.94 & 1.06 & $V_{X X}^{\text {rung }}(\mathrm{eV})$ & 0.18 & 0.16 & 0.13 & $V_{L L}(\mathrm{eV})$ & 0.23 & 0.19 & 0.17 & $K\left(\mathrm{eV} / \AA^{2}\right)$ & 6.00 & 8.00 & 8.00 \\
\hline \hline
\end{tabular}

calculations, they report that $t_{\mathrm{PtCl}}=1.54 \mathrm{eV}$ and $t_{\mathrm{PtBr}}$ $=1.30 \mathrm{eV}$ for $\left[\mathrm{Pt}(\mathrm{en})_{2} X\right],{ }^{4,38,39}$ while $t_{\mathrm{PtCl}}=1.60 \mathrm{eV}$ and $t_{\mathrm{PtBr}}$ $=1.50 \mathrm{eV}$ for $\left[\operatorname{Pt}\left(\mathrm{NH}_{3}\right)_{2} X_{3}\right] .^{40,41}$ Here, we take $t_{M X}$ to be $1.54,1.35$, and $1.32 \mathrm{eV}$ for (bpym) $[\mathrm{Pt}(\mathrm{en}) \mathrm{Cl}]_{2}, \quad$ (bpym) $\times[\mathrm{Pt}(\mathrm{en}) \mathrm{Br}]_{2}$, and (bpy) $[\mathrm{Pt}(\text { dien }) \mathrm{Br}]_{2}$, respectively, considering the consistency of the resultant theoretical findings with experimental observations.

Another essential one-body parameter, the relative on-site energy $\varepsilon_{M}-\varepsilon_{X}$, may also be less dependent on the rung ligands, but it is not so established as $t_{M X}$ even in $M X$ single chains. The Los Alamos group, on one hand, reports that $\varepsilon_{\mathrm{Cl}}=-1.32 \mathrm{eV}$ and $\varepsilon_{\mathrm{Br}}=-0.58 \mathrm{eV}$ for $\left[\mathrm{Pt}(\mathrm{en})_{2} \mathrm{X}\right],{ }^{4,38,39}$ while $\varepsilon_{\mathrm{Cl}}=-2.90 \mathrm{eV}$ and $\varepsilon_{\mathrm{Br}}=-2.30 \mathrm{eV}$ for $\left[\mathrm{Pt}\left(\mathrm{NH}_{3}\right)_{2} X_{3}\right],{ }^{40,41} \mathrm{but}$, on the other hand, suggests another possibility that $\varepsilon_{\mathrm{Cl}}=$ $-4.24 \mathrm{eV}$ and $\varepsilon_{\mathrm{Br}}=-1.20 \mathrm{eV}$ for $\left[\mathrm{Pt}(\mathrm{en})_{2} X\right],{ }^{42}$ where $\varepsilon_{M}$ is set equal to zero. Therefore, we tune the on-site energies within these estimates so as to reproduce experimental observations.

Coulomb interactions much more vary with the surrounding ligands and seriously depend on the modeling. For instance, the on-site repulsion $U_{\mathrm{Pt}}$ effectively amounts to a few $\mathrm{eV}$ in a pure Hubbard model, ${ }^{42,43}$ whereas it is strongly suppressed to a half $\mathrm{eV}$ or less in a fully extended model with power-law decaying Coulomb terms. ${ }^{44}$ Taking it into consideration that any empirical estimate of $U_{\mathrm{Pt}}$ does not exceed $2 \mathrm{eV},{ }^{13,15,45-47}$ relying upon a well established criterion $U_{\mathrm{Pt}}$ $\simeq U_{\mathrm{Br}} \lesssim U_{\mathrm{Cl}},{ }^{4,39}$ and strictly keeping the restriction that the farther, the smaller, we compare our calculations with experimental findings on an absolute scale. The thus-obtained $d-p-\pi$ model parameters for $M X$ ladders are listed in Table I. Among the Coulomb correlation parameters employed, $U_{A}$ $(A=M, X, L)$ and $V_{M X}^{\mathrm{leg}}$ play predominant roles in reproducing the main features of the optical-conductivity spectra. The rest are much less effective for the optical properties. Indeed, we have many parameters, but their output is not so adjustable as might be expected. The effect and tuning of each parameter are further discussed and visualized in the Appendix in order to demonstrate the reliability of our parametrization.

Table II claims that our theory well interprets X-ray diffraction measurements as well as optical observations. ${ }^{27,28}$ Since optical conductivity of definite polarization is proportional to the relevant interatomic separation squared [see Eqs. (4.1) and (4.2)], a direct comparison of the calculations to the bare observations gives lattice constants. The Peierls gap and the lattice distortion, which are in proportion to each other, are determined within our calculation independent of any optical measurement. The optical excitation energy $E_{\mathrm{CT}}$ is closely related but does not coincide with the Peierls gap in the present case. The consequent lattice parameters correspond to the observations within a factor of 1.4 , which guarantees our interpretation of the optical- conductivity spectra. The calculated optical gaps are also in good agreement with the observations, which justifies our parametrization. The onsite repulsion $U_{M}$ and the site-diagonal coupling constant $\beta$ competitively dominate $E_{\mathrm{CT}}$, whereas the elastic constant $K$ is decisive of $u$. A general tendency for halogen-ion displacements, $u(\mathrm{I})<u(\mathrm{Br})<u(\mathrm{Cl})$, holds in $M X$ ladders $^{28}$ as well as in $M X$ single chains. ${ }^{48}$

\section{OPTICAL-CONDUCTIVITY SPECTRA}

\section{A. Calculational procedure}

In order to discuss optical absorption as a function of the polarization of incident light $\left(\boldsymbol{E}_{\text {in }}\right)$, we define current operators along ladder legs $(\| \boldsymbol{c})$ and rungs $(\perp \boldsymbol{c})$ as

$$
\begin{aligned}
\mathcal{J}_{\|}= & \frac{i e}{\hbar} c_{M X} \sum_{l, n, s}\left[\left(t_{M X}+\alpha u_{n: l}\right) a_{n+1: l M s}^{\dagger} a_{n: l X s}\right. \\
& +\left(t_{M X}-\alpha u_{n: l}\right) a_{n: l X s}^{\dagger} a_{n: l M s}+\left(t_{X L}+\gamma u_{n: l}\right) a_{n+1: L s}^{\dagger} a_{n: l X s} \\
& \left.+\left(t_{X L}-\gamma u_{n: l}\right) a_{n: l X s}^{\dagger} a_{n: L s}-\text { H.c. }\right], \\
\mathcal{J}_{\perp}= & \frac{i e}{\hbar} c_{M L} \sum_{l, n, s}(-1)^{l}\left[t_{M L} a_{n: l M s}^{\dagger} a_{n: L s}+\left(t_{X L}-\gamma u_{n: l}\right) a_{n: l X s}^{\dagger} a_{n: L s}\right. \\
& \left.+\left(t_{X L}+\gamma u_{n: l}\right) a_{n: l X s}^{\dagger} a_{n+1: L s}-\text { H.c. }\right],
\end{aligned}
$$

where $2 c_{M X}$ and $2 c_{M L}$ are the intermetallic separations in the

TABLE II. Theoretical (bare) and experimental (inside parentheses) estimates of structural and optical parameters for (A) (bpym) $[\mathrm{Pt}(\mathrm{en}) \mathrm{Cl}]_{2}, \quad$ (B) (bpym) $[\mathrm{Pt}(\mathrm{en}) \mathrm{Br}]_{2}$, and (C) (bpy) [Pt(dien) $\mathrm{Br}]_{2} .2 c_{M X}$ and $2 c_{M L}$ correspond to the Pt- $X$-Pt and Pt- $L$-Pt distances, respectively, while $u$ to the $X$ displacement from the mid point. $E_{\mathrm{CT}}$ is the intrachain charge-transfer excitation energy.

\begin{tabular}{ccccc}
\hline \hline & $\begin{array}{c}c_{M X} \\
(\AA)\end{array}$ & $\begin{array}{c}c_{M L} \\
(\AA)\end{array}$ & $\begin{array}{c}u \\
(\AA)\end{array}$ & $\begin{array}{c}E_{\mathrm{CT}} \\
(\mathrm{eV})\end{array}$ \\
\hline $\mathrm{A}$ & $1.98(2.72)$ & $1.98(2.73)$ & $0.38(0.40)$ & $3.74(3.66)$ \\
$\mathrm{B}$ & $2.00(2.77)$ & $2.00(2.74)$ & $0.28(0.29)$ & $2.47(2.36)$ \\
$\mathrm{C}$ & $3.50(2.73)$ & $7.00(5.59)$ & $0.25(0.23)$ & $2.13(2.18)$ \\
\hline \hline
\end{tabular}



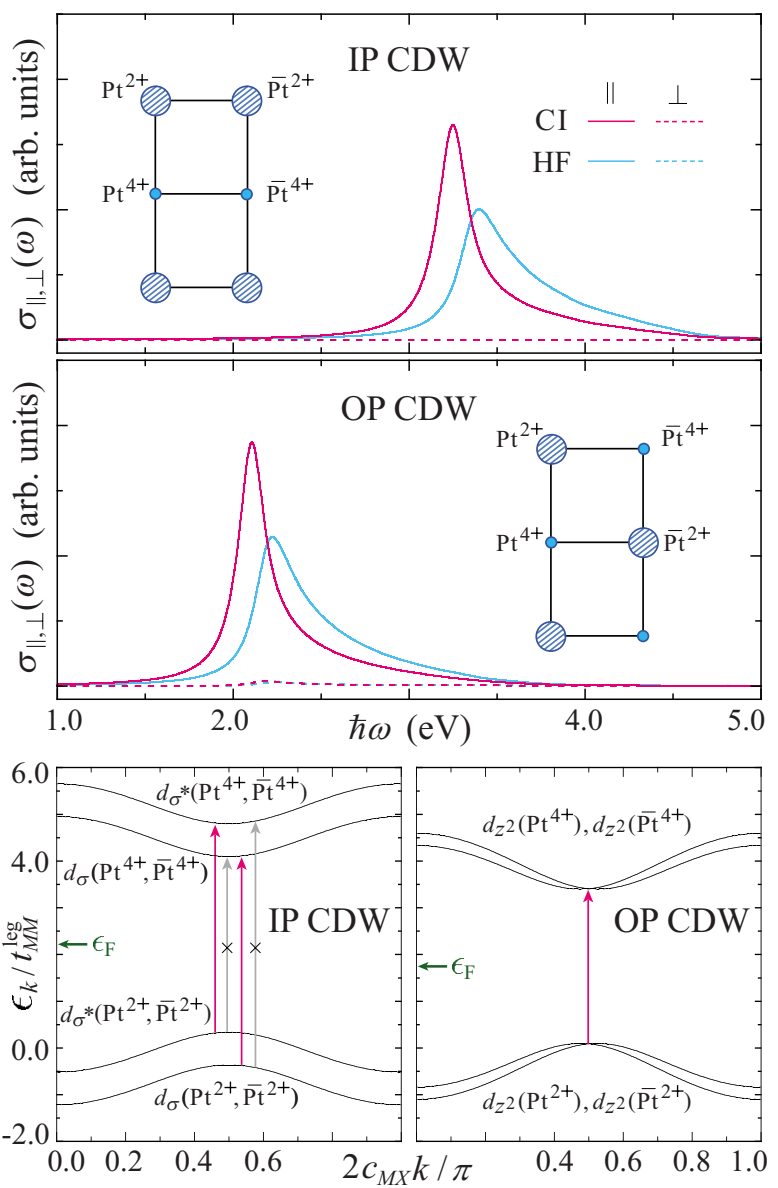

FIG. 3. (Color online) Hartree-Fock (HF) and single-excitation configuration-interaction (CI) calculations of the polarized opticalconductivity spectra parallel $(\|)$ and perpendicular $(\perp)$ to ladder legs within the single-band model, where $t_{M M}^{\mathrm{leg}}=0.75 \mathrm{eV}(0.66 \mathrm{eV})$, $t_{M M}^{\text {rung }} / t_{M M}^{\text {leg }}=0.35 \quad(0.16), \quad U_{M} / t_{M M}^{\text {leg }}=1.40 \quad(1.20), \quad V_{M M}^{\mathrm{leg}} / t_{M M}^{\mathrm{leg}}=0.35$ $(0.35), \quad V_{M M}^{\text {rung }} / t_{M M}^{\text {leg }}=0.30 \quad(0.25), \quad V_{M M}^{\mathrm{diag}} / t_{M M}^{\mathrm{leg}}=0.26 \quad(0.10), \quad$ and $\beta / \sqrt{t_{M M}^{\text {leg }} K=1.10(1.00)}$ for IP CDW (OP CDW). Hartree-Fock calculations of the relevant dispersion relations are also shown in an attempt to understand the spectral features of $\sigma_{\|}(\omega)$, where bare arrows and those with a cross attached denote major optical absorptions and optically forbidden transitions, respectively.

leg and rung directions, respectively, and are fixed at $c_{M X}$ $=c_{M L}$ and $2 c_{M X}=c_{M L}$ for (bpym) $[\mathrm{Pt}(\mathrm{en}) X]_{2}$ and (bpy) $\times[\mathrm{Pt}(\text { dien }) \mathrm{Br}]_{2}$, respectively, in our calculation. Since the charge-transfer excitation energy is of $\mathrm{eV}$ order, ${ }^{27,28}$ the system effectively lies in the ground state at room temperature. Then, the real part of the optical conductivity reads

$$
\sigma_{\|, \perp}(\omega)=\frac{\pi}{\omega} \sum_{i}\left|\left\langle E_{i}\left|\mathcal{J}_{\|, \perp}\right| E_{0}\right\rangle\right|^{2} \delta\left(E_{i}-E_{0}-\hbar \omega\right)
$$

where $\left|E_{i}\right\rangle$ is the $i$ th-lying state of energy $E_{i}$. $\left|E_{0}\right\rangle$ is defined as

$$
\left|E_{0}\right\rangle=\prod_{\epsilon_{\mu s} \leqslant \epsilon_{F}} c_{\mu+}^{\dagger} c_{\mu-}^{\dagger}|0\rangle,
$$

where $|0\rangle$ is the true electron vacuum, $\epsilon_{F}$ is the Fermi energy, and $c_{\mu s}^{\dagger}$ creates an electron of spin $s$ in the Hartree-Fock (HF)

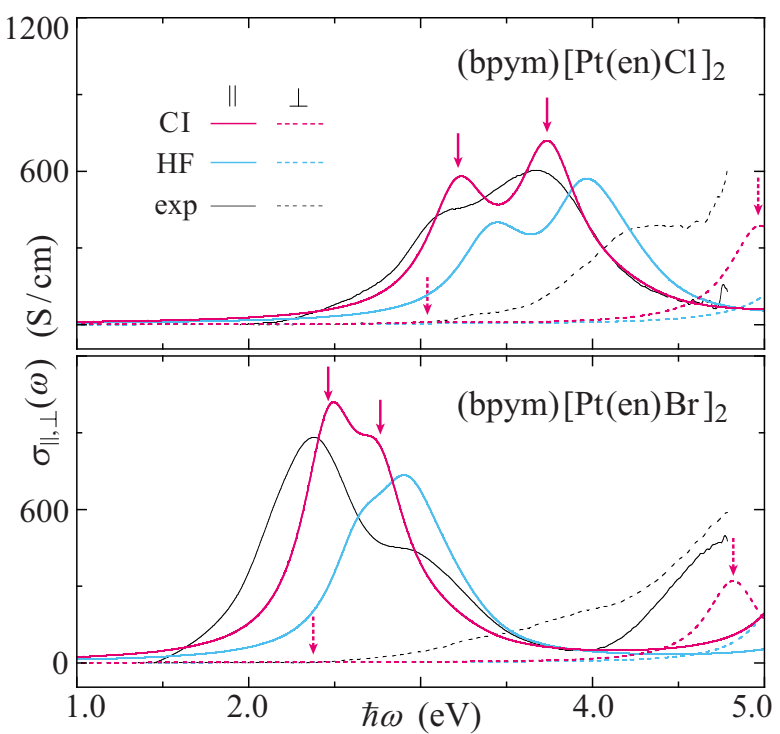

FIG. 4. (Color online) Hartree-Fock (HF) and single-excitation configuration-interaction (CI) calculations of the polarized opticalconductivity spectra parallel $(\|)$ and perpendicular $(\perp)$ to ladder legs for IP-CDW states in comparison with experimental observations (exp) of (bpym) $[\mathrm{Pt}(\mathrm{en}) X]_{2}$.

eigenstate with an eigenvalue $\epsilon_{\mu s}$. Excited states are calculated within and beyond the HF scheme, being generally defined as

$$
\left|E_{i}\right\rangle=\sum_{\epsilon_{\mu s} \leqslant \epsilon_{F}<\epsilon_{\nu s}} f(\mu, \nu, s ; i) c_{\nu s}^{\dagger} c_{\mu s}\left|E_{0}\right\rangle .
$$

Every excited state of the HF type is a single Slater determinant, where $f(\mu, \nu, s ; i)=\delta_{\mu \nu s, i}$. Those of the configurationinteraction $(\mathrm{CI})$ type consist of resonating Slater determinants, where $f(\mu, \nu, s ; i)$ satisfies

$$
\begin{aligned}
& \sum_{\epsilon_{\mu s} \leqslant \epsilon_{F}<\epsilon_{\nu s}}\left\langle E_{0}\left|c_{\mu^{\prime} s^{\prime}}^{\dagger} c_{\nu^{\prime} s^{\prime}} \mathcal{H} c_{\nu s}^{\dagger} c_{\mu s}\right| E_{0}\right\rangle f(\mu, \nu, s ; i) \\
& \quad=E_{i} f\left(\mu^{\prime}, \nu^{\prime}, s^{\prime} ; i\right),
\end{aligned}
$$

that is, the unitary matrix $f(\mu, \nu, s ; i)$ diagonalizes the original Hamiltonian $\mathcal{H}$. Since the HF Hamiltonian $\mathcal{H}_{\mathrm{HF}}$ is diagonal with respect to pure particle-hole states as $\left\langle E_{0}\left|c_{\mu^{\prime} s^{\prime}}^{\dagger} c_{\nu^{\prime} s^{\prime}} \mathcal{H}_{\mathrm{HF}} c_{\nu s}^{\dagger} c_{\mu s}\right| E_{0}\right\rangle=\delta_{\mu^{\prime} \nu^{\prime} s^{\prime}, \mu \nu s}\left(E_{0}-\epsilon_{\mu s}+\epsilon_{\nu s}\right)$, the residual component $\mathcal{H}-\mathcal{H}_{\mathrm{HF}}$ mixes the Slater determinants and reduces the interband transition energy (see Figs. 4 and 5 later). Equation (4.3) calculated is Lorentzian broadened.

\section{B. Single-band calculation}

Before analyzing experimental findings in detail, we calculate the optical conductivity in terms of the single-band Hamiltonian (2.1) in an attempt to demonstrate the indispensable halogen $p_{z}$ and ligand $\pi$ orbitals. Figure 3 shows that the optical observations of IP- and OP-CDW states are quite alike without contributive $p$ and $\pi$ electrons. The spectra in the leg direction are single peaked, whereas no significant spectral weight lies in the rung direction. 


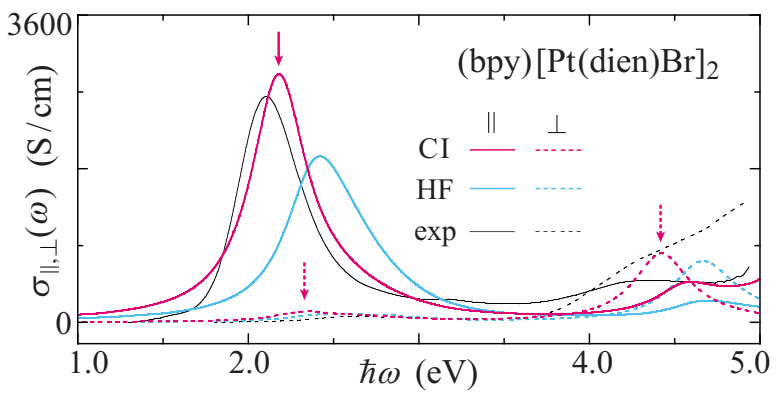

FIG. 5. (Color online) Hartree-Fock (HF) and single-excitation configuration-interaction $(\mathrm{CI})$ calculations of the polarized opticalconductivity spectra parallel $(\|)$ and perpendicular $(\perp)$ to ladder legs for an OP-CDW state in comparison with experimental observations (exp) of (bpy) $[\mathrm{Pt}(\operatorname{dien}) \mathrm{Br}]_{2}$.

Such observations are well understandable when we consider the underlying energy structures. Since the $d_{z^{2}}$ orbitals of equally valent platinum ions have the same energy and are well hybridized with each other, we find well split filled and/or conduction bands in IP CDW, while the two intrachain $d_{z^{2}}\left(\mathrm{Pt}^{2+}\right) / d_{z^{2}}\left(\mathrm{Pt}^{4+}\right)$ bands remain almost degenerate with each other in OP CDW. The pronounced peak of $\sigma_{\|}(\omega)$ is attributed to the interband excitations at the zone center. When an electron is pumped up from the filled to conduction bands, there are four types of transitions possible in general.
However, the lowest- and highest-energy ones are optically forbidden and the rest, optically allowed, cost the same energy. The conduction and filled bands are exactly symmetric with respect to the Fermi level due to the electron-hole symmetry preserved. That is why not only the OP-CDW spectrum but also the IP-CDW spectrum is single peaked. The single-peak structure remains unchanged with excitonic effect on. A consideration of $X p_{z}$ and/or $L \pi$ orbitals leads to the breakdown of the electron-hole symmetry and lifts the degeneracy between the optical observations of IP CDW and OP CDW, which is essential to the understanding of experimental findings.

The vanishing weight of $\sigma_{\perp}(\omega)$ is also due to the sleeping $p$ and $\pi$ electrons and is never in agreement with any experiment. We are thus lead to the $d-p-\pi$ modeling.

\section{C. $d-p-\pi$ description}

In Figs. 4 and 5 , we compare the $d-p-\pi$ calculations of the optical conductivity with experimental observations, ${ }^{28}$ that is, the Kramers-Kronig transforms of polarized reflectivity spectra for the single crystals at room temperature. The calculations qualitatively interpret most of the spectral features within the HF scheme and quantitatively improves with excitonic effects. We have two arguments, in particular: (i) For $\boldsymbol{E}_{\text {in }} \| \boldsymbol{c}$, the main absorption band is double peaked in (bpym) $[\mathrm{Pt}(\mathrm{en}) \mathrm{X}]_{2}$ but single peaked in (bpy) $[\mathrm{Pt}(\mathrm{dien}) \mathrm{Br}]_{2}$.

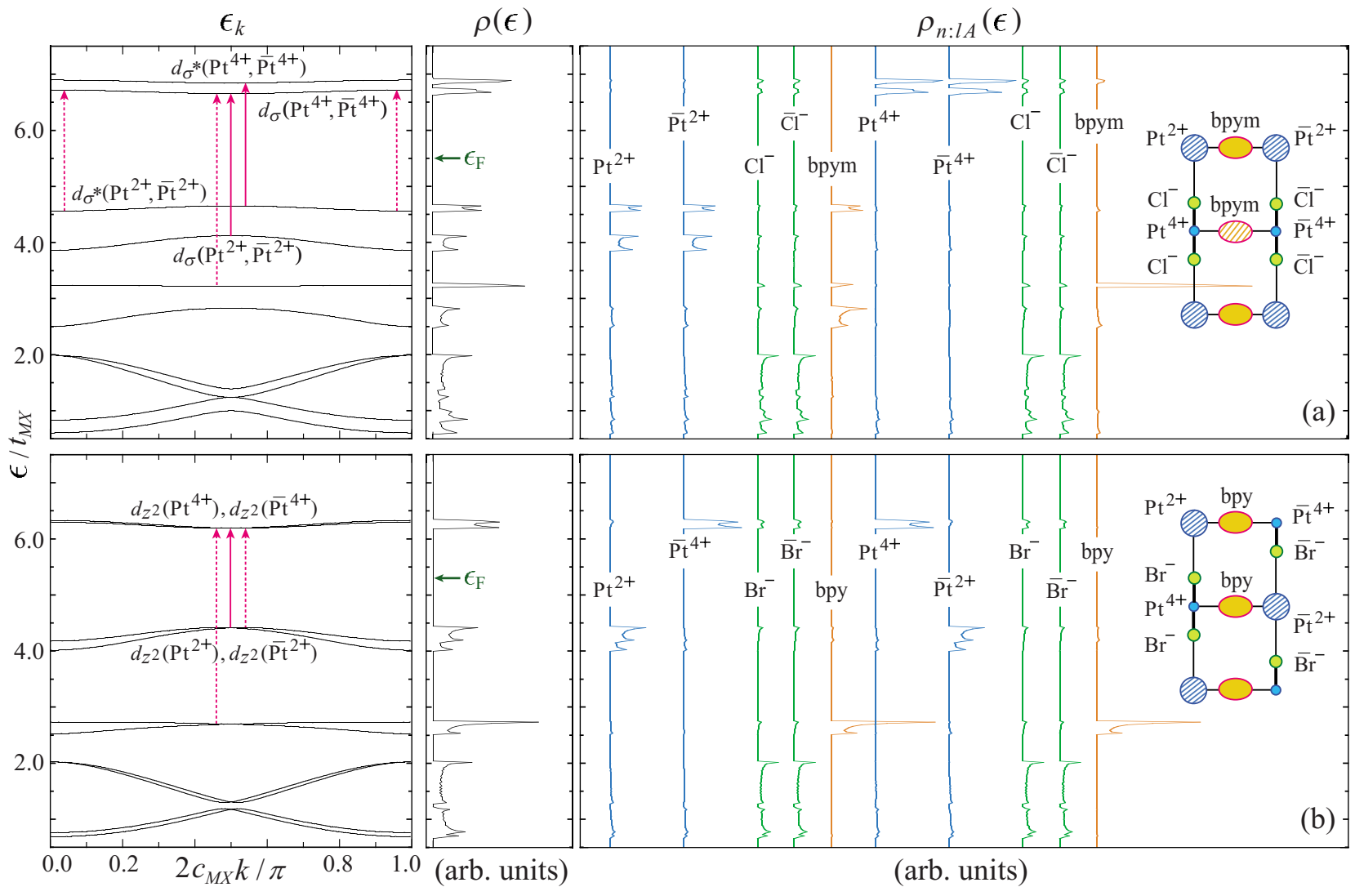

FIG. 6. (Color online) Hartree-Fock calculations of the dispersion relation $\epsilon_{k}$ and the local (total) density of states $\rho_{n: l A}(\epsilon)[\rho(\epsilon)$ $\left.\equiv \Sigma_{n, l, A} \rho_{n: l A}(\epsilon)\right]$ for the (a) IP-CDW and (b) OP-CDW states describing (bpym) $[\mathrm{Pt}(\mathrm{en}) \mathrm{Cl}]_{2}$ and (bpy) $\left[\mathrm{Pt}(\text { dien)Br }]_{2}\right.$, respectively. Major optical absorptions with $\boldsymbol{E}_{\text {in }} \| \boldsymbol{c}$ and $\boldsymbol{E}_{\text {in }} \perp \boldsymbol{c}$ are indicated by solid and dotted arrows, respectively, in Figs. 4 and 5 as well as here. 


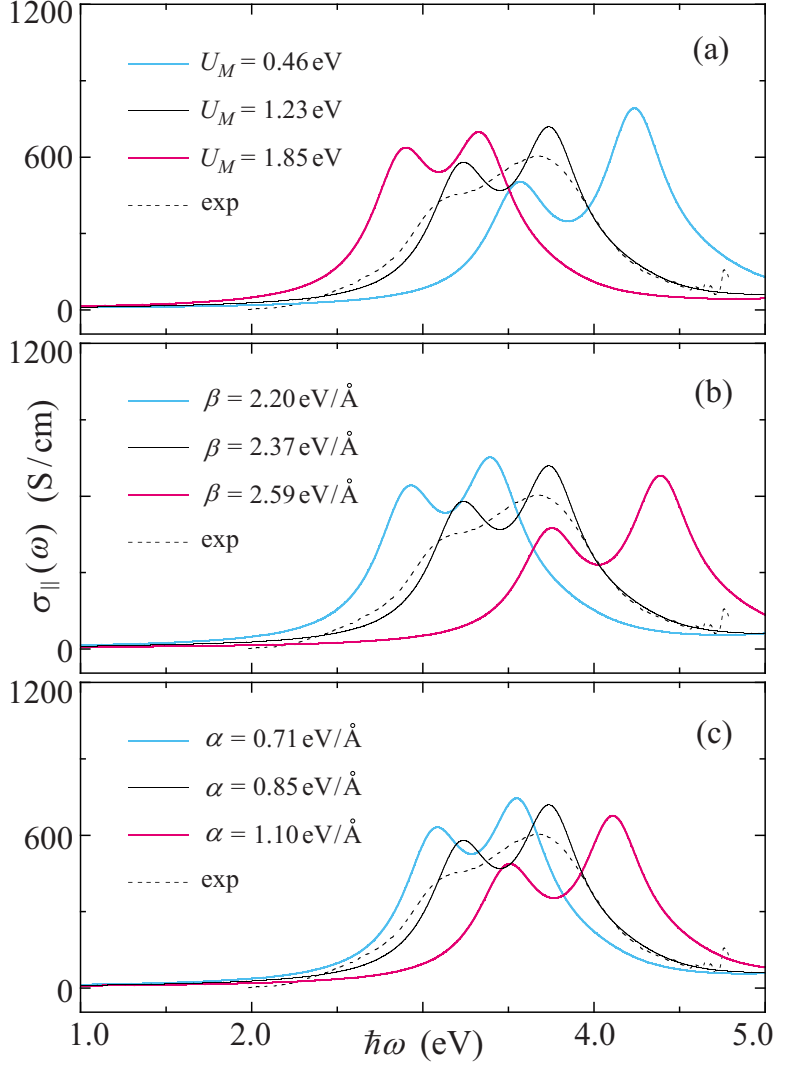

FIG. 7. (Color online) Single-excitation configurationinteraction calculations (solid lines) of the polarized opticalconductivity spectra parallel to ladder legs for IP-CDW states in comparison with an experimental observation (a dotted line) of (bpym) $[\mathrm{Pt}(\mathrm{en}) \mathrm{Cl}]_{2}$, where (a) $U_{M}$, (b) $\beta$, and (c) $\alpha$ are tuned, while the rest are fixed at set $\mathrm{A}$ in Table I.

(ii) For $\boldsymbol{E}_{\text {in }} \perp \boldsymbol{c}$, significant absorption is observed not only in (bpy) $[\mathrm{Pt}(\text { dien }) \mathrm{Br}]_{2}$ but also in (bpym) $[\mathrm{Pt}(\mathrm{en}) X]_{2}$.

$\sigma_{\|}(\omega)$ corresponding spectra measured on the $M X$ singlechain compounds $\left[\mathrm{Pt}(\mathrm{en})_{2} \mathrm{X}\right]\left(\mathrm{ClO}_{4}\right)_{2}$ are all single peaked ${ }^{49}$ at $\hbar \omega \simeq 2.7 \mathrm{eV}$ for $X=\mathrm{Cl}$ and at $\hbar \omega \simeq 2.0 \mathrm{eV}$ for $X=\mathrm{Br}$. Figure 5 is reminiscent of these observations, whereas Fig. 4 must be characteristic of the ladder system. Although (bpym) $[\mathrm{Pt}(\mathrm{en}) \mathrm{X}]_{2}$ and (bpy) $[\mathrm{Pt}(\text { dien }) \mathrm{Br}]_{2}$ are both shaped like ladders, their electronic structures are distinct from each other, as is shown in Fig. 6. In an IP-CDW state, every pair of Pt $d_{z^{2}}$ orbitals facing each other across a ligand is well hybridized and split into their bonding $\left(d_{\sigma}\right)$ and antibonding $\left(d_{\sigma^{*}}\right)$ combinations with the help of the bridging $\pi$ orbital. The local density of states reveals a significant contribution of $\pi$ orbitals to the $d_{\sigma} *$ bands. The fully occupied $d_{z^{2}}\left(\mathrm{Pt}^{2+}\right)$ orbitals are much more stabilized than the vacant $d_{z^{2}}\left(\mathrm{Pt}^{4+}\right)$ ones, that is, to say, $\varepsilon\left[d_{\sigma}^{*}\left(\mathrm{Pt}^{4+}, \overline{\mathrm{Pt}^{4+}}\right)\right]-\varepsilon\left[d_{\sigma}\left(\mathrm{Pt}^{4+}, \overline{\mathrm{Pt}^{4+}}\right)\right]$ $\ll \varepsilon\left[d_{\sigma} *\left(\mathrm{Pt}^{2+}, \overline{\mathrm{Pt}}^{2+}\right)\right]-\varepsilon\left[d_{\sigma}\left(\mathrm{Pt}^{2+}, \overline{\mathrm{Pt}}^{2+}\right)\right]$. It is the broken electron-hole symmetry that unequalizes the optically allowed excitations of two types. Thus, we find a doublepeaked absorption band. The essential $d-\pi$ hybridization is characteristic of (bpym) $[\mathrm{Pt}(\mathrm{en}) \mathrm{X}]_{2}$. In an OP-CDW state, on the other hand, there hardly occurs interchain hybridization

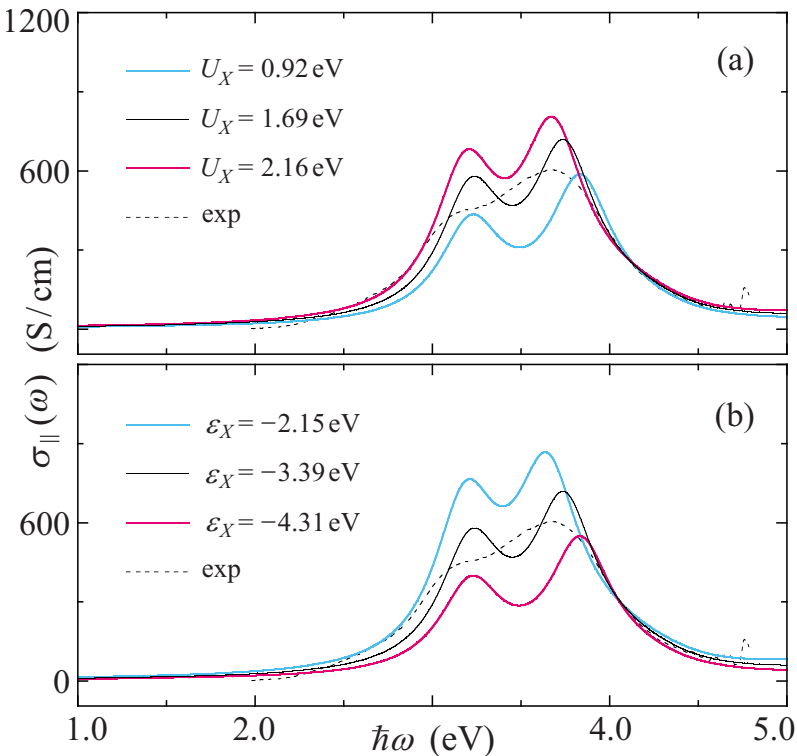

FIG. 8. (Color online) Single-excitation configurationinteraction calculations (solid lines) of the polarized opticalconductivity spectra parallel to ladder legs for IP-CDW states in comparison with an experimental observation (a dotted line) of (bpym) $[\mathrm{Pt}(\mathrm{en}) \mathrm{Cl}]_{2}$, where (a) $U_{X}$ and (b) $\varepsilon_{X}$ are tuned, while the rest are fixed at set $\mathrm{A}$ in Table I.

of $\mathrm{Pt} d_{z^{2}}$ orbitals and thus the main absorption band of $\mathrm{Pt}$ character remains single peaked. The density of states is nothing more than a simple sum of poorly mixed $d, p$, and $\pi$ orbitals. (bpy) $[\mathrm{Pt}(\text { dien }) \mathrm{Br}]_{2}$ still has a strong resemblance to conventional $M X$ single-chain compounds.

$\sigma_{\perp}(\omega)$ with the $p$ and $\pi$ electrons included, an absorption of Pt character in the rung direction is activated in an IPCDW state and is strengthened, roughly doubled, in an OPCDW state. However, it is still much less recognizable than that in the leg direction. Most of the spectral weight is distributed to the higher-energy region, which is attributable to $\pi$ - $d$ charge-transfer excitations. The single-excitation CI scheme seems still incomplete but fully demonstrates the crucial role of electronic correlations in reproducing the observations quantitatively. It may also be effective to take ligand $\pi^{*}$ orbitals into calculation. Here, we have discarded the vacant $\pi^{*}$ orbitals, on one hand, assuming them to be higher lying than $\mathrm{Pt} d_{z^{2}}$ orbitals and, on the other hand, avoiding further increase of the number of parameters. A pioneering density-functional study ${ }^{37}$ on (bpym) $[\mathrm{Pt}(\mathrm{en}) \mathrm{Cl}]_{2}$ proposes a level scheme of the bpym $\pi^{*}$ orbitals being sandwiched between the $d_{z^{2}}\left(\mathrm{Pt}^{2+}\right)$ and $d_{z^{2}}\left(\mathrm{Pt}^{4+}\right)$ bands. Such a scenario looks consistent with our underestimation of $\sigma_{\perp}(\omega)$ for (bpym) $[\mathrm{Pt}(\mathrm{en}) \mathrm{X}]_{2}$ and may explain the low-energy shoulder or foot of its widespread band. On the other hand, the bpy $\pi^{*}$ orbitals are likely to lie above the Pt $d_{z^{2}}$ bands, judging from Fig. 5.

\section{SUMMARY}

(bpym) $[\operatorname{Pt}(\mathrm{en}) X]_{2}$ reveal themselves as $d$ - $p$ - $\pi$-hybridized multiband ladder materials with a ground state of the IP- 


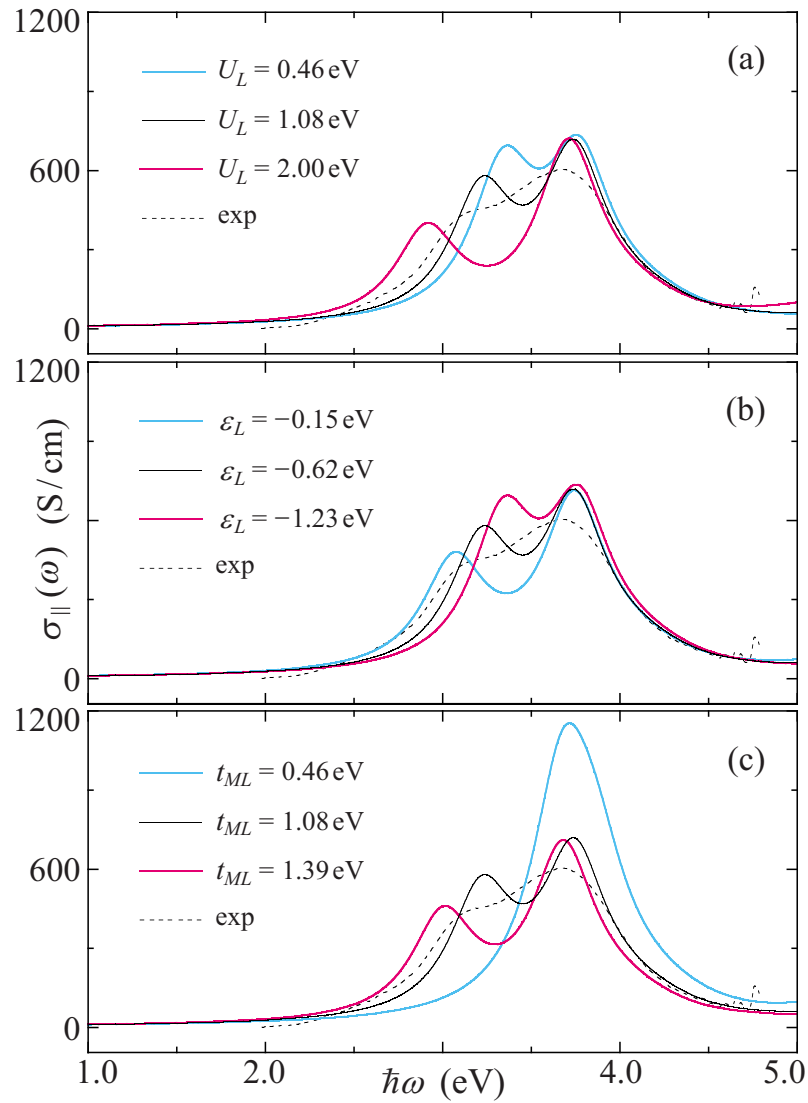

FIG. 9. (Color online) Single-excitation configurationinteraction calculations (solid lines) of the polarized opticalconductivity spectra parallel to ladder legs for IP-CDW states in comparison with an experimental observation (a dotted line) of (bpym) $[\mathrm{Pt}(\mathrm{en}) \mathrm{Cl}]_{2}$, where (a) $U_{L}$, (b) $\varepsilon_{L}$, and (c) $t_{M L}$ are tuned, while the rest are fixed at set $\mathrm{A}$ in Table I.

CDW type, while (bpy) $[\mathrm{Pt}(\text { dien }) \mathrm{Br}]_{2}$ as a $d_{z^{2} \text {-single-band }}$ double-chain material with a ground state of the OP-CDW type, which is reminiscent of conventional $M X$ chain compounds. The two ground states are highly competitive and both materials sit in the vicinity of the phase boundary. An iodine derivative of the former compounds, ( $\mu$-bpym) $\times[\mathrm{Pt}(\mathrm{en}) \mathrm{I}]_{2} \mathrm{I}_{4} \cdot 2 \mathrm{H}_{2} \mathrm{O},{ }^{28}$ might have a ground state of OP$\mathrm{CDW}$ character. ${ }^{50}$

There lie ahead fascinating topics such as quantum phase transitions and nonlinear photoproducts in this geometrically designed Peierls-Hubbard multiband system. Palladium and nickel analogs as well as ligand substitution will contribute toward realizing further density-wave states ${ }^{31}$ possible in a multiorbital ladder lattice. Photogenerated excitons and their relaxation channels were extensively calculated for $M X$ (Refs. 1, 2, 51, and 52) and $M M X$ (Refs. 53 and 54) chains, and the predicted scenarios were indeed demonstrated experimentally. ${ }^{48,55}$ Photoexcited $M X$ ladders are more and more interesting. Contrastive materials with IP-CDW and OP-CDW backgrounds have been provided and identified. The next stage is ready for further investigations.

\section{ACKNOWLEDGMENTS}

We are grateful to K. Iwano for fruitful discussions and

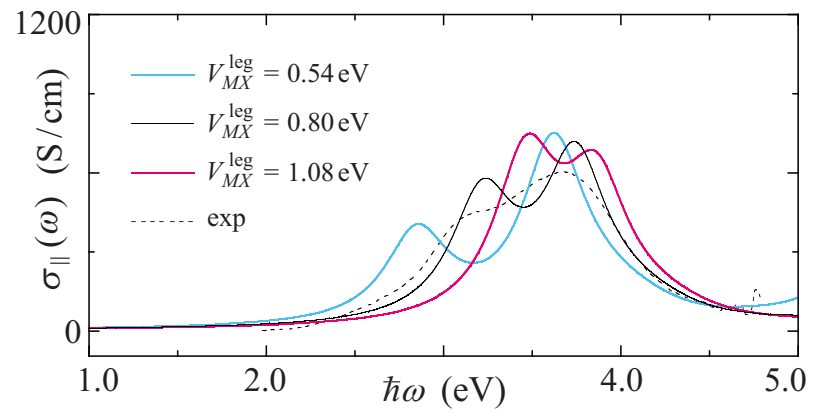

FIG. 10. (Color online) Single-excitation configurationinteraction calculations (solid lines) of the polarized opticalconductivity spectra parallel to ladder legs for IP-CDW states in comparison with an experimental observation (a dotted line) of (bpym) $[\mathrm{Pt}(\mathrm{en}) \mathrm{Cl}]_{2}$, where all the parameters but $V_{M X}^{\mathrm{leg}}$ are fixed at set A in Table I.

valuable comments on our calculation. $\mathrm{H}$. Matsuzaki and $\mathrm{H}$. Okamoto have allowed and encouraged us to discuss their elaborate optical observations. Their kindness is greatly appreciated. We further thank D. Kawakami, M. Yamashita, A. Kobayashi, and H. Kitagawa for useful information on their brand-new $M X$ ladder products. This work was supported by the Ministry of Education, Culture, Sports, Science and Technology of Japan.

\section{APPENDIX: ON THE PARAMETER TUNING}

We have reached the best solutions in Figs. 4 and 5 systematically tuning all the parameters. The opticalconductivity spectral shape monotonically varies as we tune each parameter. We demonstrate the parameter tuning for (bpym) $[\mathrm{Pt}(\mathrm{en}) \mathrm{Cl}]_{2}$ and discuss what roles leading parameters play in reproducing the spectra.

Varying $U_{M}$ slides, rather than deform, the spectrum (Fig. 7). With increasing $U_{M}$, the Peierls gap is reduced and any charge-transfer excitation energy monotonically decreases. The effect of electron-lattice interactions can be understood in the same context. The site-diagonal coupling constant $\beta$ straightforwardly stabilizes a CDW on metal sites. Considering $M-X$ charge-transfer energy gains, the site-off-diagonal coupling constant $\alpha$ also stabilizes a site-diagonal CDW rather than a site-off-diagonal (bond-centered) CDW, provided $\varepsilon_{M} \neq \varepsilon_{X}$. Both $\alpha$ and $\beta$ work against $U_{M}$. All these parameters position the intrachain charge-transfer band.

$U_{X}$ and $\varepsilon_{X}$ adjust the spectral weight of the main absorption band originating from intrachain $M-X$ charge transfer excitations (Fig. 8). The oscillator strength of the chargetransfer band increases with activated $p$ electrons. Increasing $U_{X}$ induces oxidation of $X^{-}$ions, while $\varepsilon_{X}$ approaching to $\varepsilon_{M}$ activates $d$ - $p$ hybridization. The spectral weight increases with increasing $U_{X}$ and decreasing $\varepsilon_{M}-\varepsilon_{X}$.

Parameters related to rung ligands control the structure of the main absorption band (Fig. 9). With increasing $U_{L}, \varepsilon_{L}$ approaching to $\varepsilon_{M}$, and increasing $t_{M L}, d$ - $\pi$ hybridization is encouraged. Then, the intrachain $d$ bands of $\mathrm{Pt}^{2+}$ character split into their bonding and antibonding combinations, and the charge-transfer band is doubly peaked. 
Finally we take a look at the effect of different-site Coulomb interactions (Fig. 10). The HF decomposition of any Coulomb term reminds us that the Coulomb interaction originates from electron hopping between the relevant sites. $V_{M X}^{\mathrm{leg}}$ indeed modulates the band gap in the same way as $\alpha$ at the HF level. However, the configuration interaction restructures the charge-transfer band and drastically changes its double-peaked features. With $V_{M X}^{\mathrm{leg}}$ large enough, the lower-energy absorption can even be stronger than the higher-energy one.

$V_{M M}^{\mathrm{rung}}$ and $V_{M M}^{\mathrm{diag}}$ are also important Coulomb interactions, though they act on the next-nearest-neighbor sites. They highly compete with each other for the ground-state valence arrangement. Therefore, these parameters are much less tunable and determined with smaller uncertainty. Thus, we are led to the parametrization in Table I and theoretical findings in Figs. 4 and 5. Considering the structural data as well, there is no better solution within the present modeling.
${ }^{1}$ A. Mishima and K. Nasu, Phys. Rev. B 39, 5758 (1989).

${ }^{2}$ A. Mishima and K. Nasu, Phys. Rev. B 39, 5763 (1989).

${ }^{3}$ J. T. Gammel, A. Saxena, I. Batistić, A. R. Bishop, and S. R. Phillpot, Phys. Rev. B 45, 6408 (1992).

${ }^{4}$ S. M. Weber-Milbrodt, J. T. Gammel, A. R. Bishop, and E. Y. Loh, Jr., Phys. Rev. B 45, 6435 (1992).

${ }^{5}$ B. M. Craven and D. Hall, Acta Crystallogr. 14, 475 (1961).

${ }^{6} \mathrm{H}$. Toftlund and O. Simonsen, Inorg. Chem. 23, 4261 (1984).

${ }^{7}$ K. Toriumi, Y. Wada, T. Mitani, S. Bandow, M. Yamashita, and Y. Fujii, J. Am. Chem. Soc. 111, 2341 (1989).

${ }^{8}$ K. Iwano, Phys. Rev. B 70, 241102(R) (2004).

${ }^{9}$ H. Matsuzaki, M. Yamashita, and H. Okamoto, J. Phys. Soc. Jpn. 75, 123701 (2006).

${ }^{10}$ K. Marumoto, H. Tanaka, S. Kuroda, T. Manabe, and M. Yamashita, Phys. Rev. B 60, 7699 (1999).

${ }^{11}$ H. Matsuzaki, K. Iwano, T. Aizawa, M. Ono, H. Kishida, M. Yamashita, and H. Okamoto, Phys. Rev. B 70, 035204 (2004).

${ }^{12}$ A. Mishima and K. Nasu, Phys. Rev. B 40, 5593 (1989).

${ }^{13}$ M. Haruki and S. Kurita, Phys. Rev. B 39, 5706 (1989).

${ }^{14}$ S. Yamamoto, Phys. Lett. A 247, 422 (1998).

${ }^{15}$ G. S. Kanner, J. T. Gammel, S. P. Love, S. R. Johnson, B. Scott, and B. I. Swanson, Phys. Rev. B 50, 18682 (1994).

${ }^{16}$ S. Yamamoto, Phys. Rev. B 63, 125124 (2001).

${ }^{17}$ M. Kuwabara and K. Yonemitsu, J. Mater. Chem. 11, 2163 (2001).

${ }^{18}$ B. I. Swanson, M. A. Stroud, S. D. Conradson, and M. H. Zietlow, Solid State Commun. 65, 1405 (1988).

${ }^{19}$ S. Yamamoto, Phys. Rev. B 64, 140102(R) (2001).

${ }^{20}$ S. Yamamoto, J. Phys. Chem. Solids 63, 1489 (2002).

${ }^{21}$ H. Matsuzaki, T. Matsuoka, H. Kishida, K. Takizawa, H. Miyasaka, K. Sugiura, M. Yamashita, and H. Okamoto, Phys. Rev. Lett. 90, 046401 (2003).

${ }^{22}$ K. Yonemitsu and N. Miyashita, Phys. Rev. B 68, 075113 (2003).

${ }^{23}$ H. Kitagawa, N. Onodera, T. Sonoyama, M. Yamamoto, T. Fukawa, T. Mitani, M. Seto, and Y. Maeda, J. Am. Chem. Soc. 121, 10068 (1999).

${ }^{24}$ S. Yamamoto, J. Phys. Soc. Jpn. 70, 1198 (2001).

${ }^{25}$ K. Sakai, Y. Tanaka, Y. Tsuchiya, K. Hirata, T. Tsubomura, S. Iijima, and A. Bhattacharjee, J. Am. Chem. Soc. 120, 8366 (1998)

${ }^{26}$ J. Yi, T. Miyabayashi, M. Ohashi, T. Yamagata, and K. Mashima, Inorg. Chem. 43, 6596 (2004).

${ }^{27}$ A. Kobayashi and H. Kitagawa, J. Am. Chem. Soc. 128, 12066 (2006)

${ }^{28}$ D. Kawakami, M. Yamashita, S. Matsunaga, S. Takaishi, T. Kajiwara, H. Miyasaka, K. Sugiura, H. Matsuzaki, and H. Okamoto,
Angew. Chem. 118, 7372 (2006).

${ }^{29}$ S. Nishimoto, E. Jeckelmann, and D. J. Scalapino, Phys. Rev. B 66, 245109 (2002).

${ }^{30}$ M. Aichhorn, M. Hohenadler, E. Ya. Sherman, J. Spitaler, C. Ambrosch-Draxl, and H. G. Evertz, Phys. Rev. B 69, 245108 (2004).

${ }^{31}$ K. Funase and S. Yamamoto, J. Phys. Soc. Jpn. 75, 044717 (2006).

${ }^{32}$ L. Degiorgi, P. Wachter, M. Haruki, and S. Kurita, Phys. Rev. B 40, 3285 (1989).

${ }^{33}$ K. Nasu, J. Phys. Soc. Jpn. 52, 3865 (1983).

${ }^{34}$ D. Baeriswyl and A. R. Bishop, J. Phys. C 21, 339 (1988).

${ }^{35}$ Y. Tagawa and N. Suzuki, J. Phys. Soc. Jpn. 64, 1800 (1995).

${ }^{36}$ S. Yamamoto, Phys. Rev. B 66, 165113 (2002).

${ }^{37}$ K. Iwano and Y. Shimoi, J. Phys. Soc. Jpn. 76, 063708 (2007).

${ }^{38}$ R. C. Albers, Synth. Met. 29, 169 (1989).

${ }^{39}$ R. C. Albers, M. Alouani, J. M. Wills, and M. Springborg, Synth. Met. 42, 2739 (1991).

${ }^{40}$ M. Alouani, R. C. Albers, J. M. Wills, and M. Springborg, Phys. Rev. Lett. 69, 3104 (1992).

${ }^{41}$ M. Alouani, J. W. Wilkins, R. C. Albers, and J. M. Wills, Phys. Rev. Lett. 71, 1415 (1993).

${ }^{42}$ A. Saxena, Z. Shuai, J. T. Gammel, I. Batistić, M. Alouani, J. L. Brédas, and A. R. Bishop, Synth. Met. 71, 1659 (1995).

${ }^{43}$ S. D. Conradson, M. A. Stroud, M. H. Zietlow, B. I. Swanson, D. Baeriswyl, and A. R. Bishop, Solid State Commun. 65, 723 (1988).

${ }^{44}$ I. Batistić, X. Z. Huang, A. R. Bishop, and A. Saxena, Phys. Rev. B 48, 6065 (1993).

${ }^{45}$ S. Kurita, M. Haruki, and K. Miyagawa, J. Phys. Soc. Jpn. 57, 1789 (1988).

${ }^{46}$ Y. Wada, T. Mitani, K. Toriumi, and M. Yamashita, J. Phys. Soc. Jpn. 58, 3013 (1989).

${ }^{47}$ M. Sakai, N. Kuroda, and Y. Nishina, Phys. Rev. B 40, 3066 (1989).

${ }^{48}$ H. Okamoto and M. Yamashita, Bull. Chem. Soc. Jpn. 71, 2023 (1998)

${ }^{49}$ Y. Wada, T. Mitani, M. Yamashita, and T. Koda, J. Phys. Soc. Jpn. 54, 3143 (1985).

${ }^{50}$ D. Kawakami and M. Yamashita (private communication).

${ }^{51}$ M. Suzuki and K. Nasu, Phys. Rev. B 45, 1605 (1992).

${ }^{52}$ K. Iwano, J. Phys. Soc. Jpn. 66, 1088 (1997).

${ }^{53}$ J. Ohara and S. Yamamoto, J. Phys. Soc. Jpn. 74, 250 (2005).

${ }^{54}$ J. Ohara and S. Yamamoto, Phys. Rev. B 73, 045122 (2006).

${ }^{55}$ H. Tanaka, S. Kuroda, T. Yamashita, M. Mitsumi, and K. Toriumi, J. Phys. Soc. Jpn. 72, 2169 (2003). 